\title{
DESIGN PROCEDURE FOR TUNABLE NARROW-BAND DIGITAL FILTERS
}

\author{
Dr. Fawzy Ibrahim *
}

\begin{abstract}
A new design technique is presented for narrow-band digital notch filters. The transversal filters have linear phase and their design is based on minimization of the integrated error energy over designated approximation bands. The optimal design is formulated as a constrained quadratic programming problem. A closed-from expression for the filter coefficients is presented. The notch frequency and null depth are the tunable parameters and the tuning process is described. It is shown that tuning the filter requires a relatively minimal number of operations, a matrixvector multiply is required to change the filter coefficients. Several examples are included which show the effectiveness of the proposed design technique.
\end{abstract}

\section{INTRODUCTION}

Many applications in digital signal processing require the elimination of narrow-band interference in systems where the signal phase response is important [1,2]. Examples include radar systems, communications systems, medical applications, and speech signal processing. The weighting function for an antenna array can also be designed to reject signals at specified directions. In this case the interference is present at a single direction or at several direction, and it is important to use a linear phase filter to eliminate the interference.

A technique for designing narrow-band FIR notch filters with exactly linear phase is described. The design is formulated as a quadratic programming problem where the pass-band and stopband error energy are minimized. A closed-form expression for the optimal solution is obtained. The filter notch frequency and attenuation appear as tunable parameters in the closed-form solution. Changes in the notch frequency or attenuation can be made without additional optimization, hence, reducing the computational complexity of the tuning process. The design process described here is for single notch filters. Filters with any number of notches may be designed by an extension of this technique.

The frequency response for an ideal notch filter is given by

$$
H_{d}\left(e^{j \omega}\right)= \begin{cases}1 ; & \omega \neq \omega_{n} \\ 0 ; & \omega=\omega_{n}\end{cases}
$$

Department of specialized Electrical Engineering Military Technical College Cairo. Egypt 
where $\omega_{\mathrm{n}}$ is the notch frequency in radians/sample. For situations where it is important to reduce the effects of aliasing and noise, a pass-band and stop-band structure may be employed. The ideal response then becomes

$$
H_{d}\left(e^{j \omega}\right)= \begin{cases}1 ; & |\omega| \leq \Omega_{p} \\ 0 ; & \omega=\omega_{\mathrm{n}} \\ 0 ; & \Omega_{\mathrm{s}} \leq \omega \leq 2 \pi-\Omega_{\mathrm{s}}\end{cases}
$$

where the pass-band edge frequency is $\Omega_{\mathrm{p}}$ and the stop-band edge frequency is $\Omega_{\mathrm{s}}$ in radians / sample. If $\Omega_{\mathrm{p}}=\Omega_{\mathrm{s}}=\pi$, then the two ideal frequency responses above are identical.

\section{DIGITAL FILTER DESIGN}

To obtain an FIR linear phase approximation to the ideal notch filter frequency response, we employ a Case 1 or Case 2 filter of length L [3]. In case 1, filters have odd length and in case 2 filters have even length. The filter impulse response is symmetrical and is given by the sequence $h(n) ; n=0, \ldots, L-1$. The zero phase frequency response for these filters can be expressed as

$$
H_{0}\left(e^{j \omega}\right)= \begin{cases}\sum_{n=0}^{(L-1) / 2} a(n) \cos n \omega & ; \text { Case } 1 \\ \sum_{n=1}^{L / 2} b(n) \cos (n-1 / 2) \omega ; \text { Case } 2\end{cases}
$$

For Case 1 filters we have

$$
\begin{aligned}
& a(0)=h\left(\frac{L-1}{2}\right) \\
& a(n)=2 h\left(\frac{L-1}{2}-n\right) ; n=1,2, \ldots, \frac{L-1}{2}
\end{aligned}
$$

and For Case 2 filters

$$
b(n)=2 h\left(\frac{L}{2}-n\right) ; n=1,2 \ldots, \frac{L}{2}
$$

Using the matrix notation to formulate the problem, we define the filter coefficient vector as 


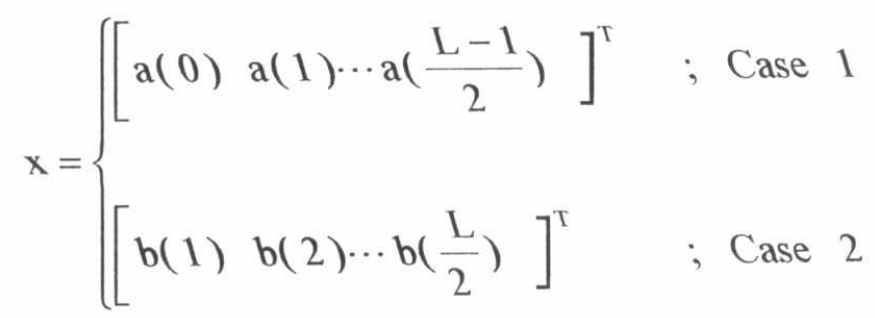

and the Fourier transform kernel vector as

$$
s(\omega)=\left\{\begin{array}{lll}
{\left[\begin{array}{ccc}
1 & \cos \omega & \cdots \cos \left(\frac{L-1}{2}\right) \omega
\end{array}\right]^{\mathrm{T}} \quad ; \text { Case } 1} \\
{\left[\begin{array}{ll}
\cos \frac{1}{2} \omega & \cos \frac{3}{2} \omega \cdots \cos \left(\frac{\mathrm{L}}{2}-\frac{1}{2}\right) \omega
\end{array}\right]^{\mathrm{T}} \quad ; \text { Case } 2}
\end{array}\right.
$$

Then, the zero phase frequency response can be written as the vector inner product

$$
H \cdot\left(e^{j \omega}\right)=x^{T} s(\omega) \text {. }
$$

The passband error energy is given by

$$
E_{p}=\frac{1}{2 \pi} \int_{-\Omega p}^{\Omega p p}\left|H_{d}\left(e^{j w}\right)-H_{0}\left(e^{j w}\right)\right|^{2} d w
$$

and can be expressed in matrix notation as

$$
E_{p}=\frac{1}{2 \pi} x^{T} Q_{p} x+b^{T} x+d
$$

where the positive definite, symmetric matrix

$$
Q_{p}=\int_{-\Omega_{p}}^{\Omega_{p}} s(\omega) S^{T}(\omega) d \omega
$$

the vector $b$ is defined as 


$$
\begin{aligned}
b & =-\frac{1}{\pi} \int_{-\Omega_{p}}^{\Omega_{p}} H_{d}\left(e^{j \omega}\right) s(\omega) d \omega \\
& =-\frac{1}{\pi} \int_{-\Omega_{p}}^{\Omega_{p}} s(\omega) d \omega
\end{aligned}
$$

and the scalar $\mathrm{d}$ is given by

$$
\begin{aligned}
d & =\frac{1}{2 \pi} \int_{-\Omega_{p}}^{\Omega_{p}} H_{d}^{2}\left(e^{j \omega}\right) d \omega \\
& =\frac{1}{\pi} \Omega_{p}
\end{aligned}
$$

(the scalar $d$ has no effect on the solution in the following optimization procedure ). Similarly, for the error energy we find

$$
\begin{aligned}
E_{s} & =\frac{1}{2 \pi} \int_{-\Omega}^{2 \pi-\Omega_{s}}\left|H_{\circ}\left(e^{j \omega}\right)\right|^{2} d \omega \\
& =\frac{1}{2 \pi} x^{T} Q_{s} x
\end{aligned}
$$

where the stop-band energy matrix

$Q_{s}=\frac{1}{2 \pi} \int_{-\Omega}^{2 \pi-\Omega_{p}} s(\omega) s^{\mathrm{T}}(\omega) d \omega$

The total error energy is

$$
\begin{aligned}
E & =E_{p}+E_{s} \\
& =\frac{1}{2 \pi} x^{T} Q x+b^{T} x+d
\end{aligned}
$$

where

$$
\mathrm{Q}=\mathrm{Qp}_{\mathrm{p}}+\mathrm{Q}_{\mathrm{S}}
$$

The zero phase frequency response is required to have magnitude $k$ at the notch frequency $\omega_{n}$, i.e.,

$$
H_{\circ}\left(e^{j \omega_{n}}\right)=k
$$

which implies that

$$
c^{\top} x=k
$$


where the column vector $\mathrm{c}$ is given by

$$
\mathrm{c}=\mathrm{s}\left(\omega_{\mathrm{n}}\right) \text {. }
$$

We can now formulate the minimization problem as a quadratic programming problem with a linear equality constraint

$$
\begin{array}{ll}
\text { Minimize } & \mathrm{E}=\frac{1}{2 \pi} \mathrm{x}^{\mathrm{T}} \mathrm{Q} \mathrm{x}+\mathrm{b}^{T} \mathrm{x} \\
\text { subject to } & c^{T} x=k
\end{array}
$$

The closed-form solution to the problem is obtained by the method of Lagrange multipliers [4] and is found to be

$$
\mathrm{X}=\mathrm{Q}^{-1} \mathrm{c}\left(\mathrm{c}^{\mathrm{T}} \mathrm{Q}^{-1} \mathrm{c}\right)^{-1} \mathrm{k}+\pi \mathrm{Q}^{-1}\left[\mathrm{c}\left(\mathrm{c}^{\mathrm{T}} \mathrm{Q}^{-1} \mathrm{c}\right)^{-1} \mathrm{c}^{\mathrm{T}} \mathrm{Q}^{-1}-\mathrm{I}\right] \mathrm{b}
$$

Note that the optimal solution for the filter coefficients is a sum of two terms. The first term depends on the prescribed null depth $k$ and the notch frequency $\omega_{n}$. The second term also involves $\mathrm{c}$ which is given by Eq. (21). Tuning the filter involves adjusting the parameters $k$ and $\omega_{n}$.

\section{NOTCH FILTER TUNING}

The notch filter can be tuned by changing the notch frequency and attenuation. Since $\mathrm{c}$ is a column vector, the term $c^{\mathrm{T}} \mathrm{Q}^{-1} \mathrm{c}$ appearing in the optimal solution is a scalar. Identifying the vectors $\hat{\mathrm{x}}$ and $\tilde{\mathrm{x}}$ as solutions to the systems

$$
\begin{aligned}
& \frac{1}{2 \pi} Q \hat{x}=c \\
& -\frac{1}{2 \pi} Q \tilde{x}=b,
\end{aligned}
$$

the optimal solution is written as

$$
\mathrm{x}=\tilde{\mathrm{x}}+\mathrm{K} \hat{\mathrm{x}}
$$

where the scalar $\mathrm{K}$ is given by

$$
K=\frac{k-c^{T} \tilde{x}}{c^{T} \hat{x}}
$$


For an infinitely deep null $k=0$ and the optimal filter coefficient vector is

$$
x=\tilde{x}-\left(\frac{c^{T} \tilde{x}}{c^{T} \hat{x}}\right) \hat{x}
$$

The first term $\tilde{\mathrm{x}}$ does not depend on $\omega_{\mathrm{n}}$. The second term is a function of the null frequency and can be updated with changing $\omega_{\mathrm{n}}$. Assuming that we have pre computed $Q^{-1}$, tuning the notch to a specific frequency $\omega_{n}$ requires the following operations

1) Find $c=s\left(\omega_{n}\right)$

2) Compute $\hat{x}=\pi Q^{-1} c$

3) Determine $K=\left(k-c^{\top} \tilde{x}\right) / c^{\top} \hat{x}$

4) Compute $x=\tilde{x}+K \hat{x}$.

The computations indicted above can be implemented as vector operations. Since the required matrix inverse is precomputed, the most computationally intensive in step 2), which requires a matrix multiplication, is reduced.

\section{DESIGN EXAMPLES}

Several examples are provided which demonstrate the effectiveness of this design technique. The first example is a narrow-band notch filter with $L=61, F_{s}=F_{p}=0.5$, and $F_{n}=0.375$ cycles/sample. The null is taken to be infinitely deep, i.e., $\mathrm{k}=0$. The magnitude response for this notch filter is shown in Figure 1. The filter has exactly linear phase. The second linear phase filter has magnitude response shown in Figure 2. Its design incorporates a pass-band configuration. In this case, $L=60, \mathrm{~F}_{\mathrm{p}}=0.4, \mathrm{~F}_{\mathrm{s}}=045$ and $\mathrm{Fn}=0.125$ cycles $/$ sample. The stopband has been included to reduce the effects of aliasing and noise encountered in some applications.

\section{CONCLUSION}

A new approach to the design of tunable notch filters is described. The FIR filters have exactly linear phase and their design may incorporate stop-bands. A stop-band is used to reduce the effects of noise and aliasing. The filter design problem is stated as quadratic programming problem and its closed-from solution is presented.

The optimized filter notch frequency and attenuation are shown to be tunable parameters. The tuning process is described and the computational advantages of this approach are noted. It is shown that tuning the filter does not require a new optimization to be carried out. Tuning requires a matrix-vector multiplication where the square matrix dimension is approximately one half the filter impulse response length. Several design examples are provided to show the effectiveness of the design technique. 


\section{REFERENCES}

[1] B. Widrow et al ,"Adaptive Noise Canceling : principles and Applications, , Proceedings of the IEEE, Vol. 63, No. 12, Dec. 1975, pp. 1692-1716.

[2] I. Kozlov and T. Mironova ,"Effectiveness of Tunable Digital Notch Filters In An MTI Systems "Radio electronics nd Communications Systems, " Vol. 28, No. 2, 1985, pp. 1215.

[3] L. Rabiner and B. Gold ,Theory And Application Of Digital Signal Processing, Englewood Cliffs, New Jersey ,Prentice-Hall ,1975.

[4] G.W. Medlin and J. W. Adams, "A New Design Technique For Maximally Linear Differentiates, "Proceedings of the International Conference on Acoustics, Speech and Signal Processing, Glasgow, May, 1989, pp. 825-828. 


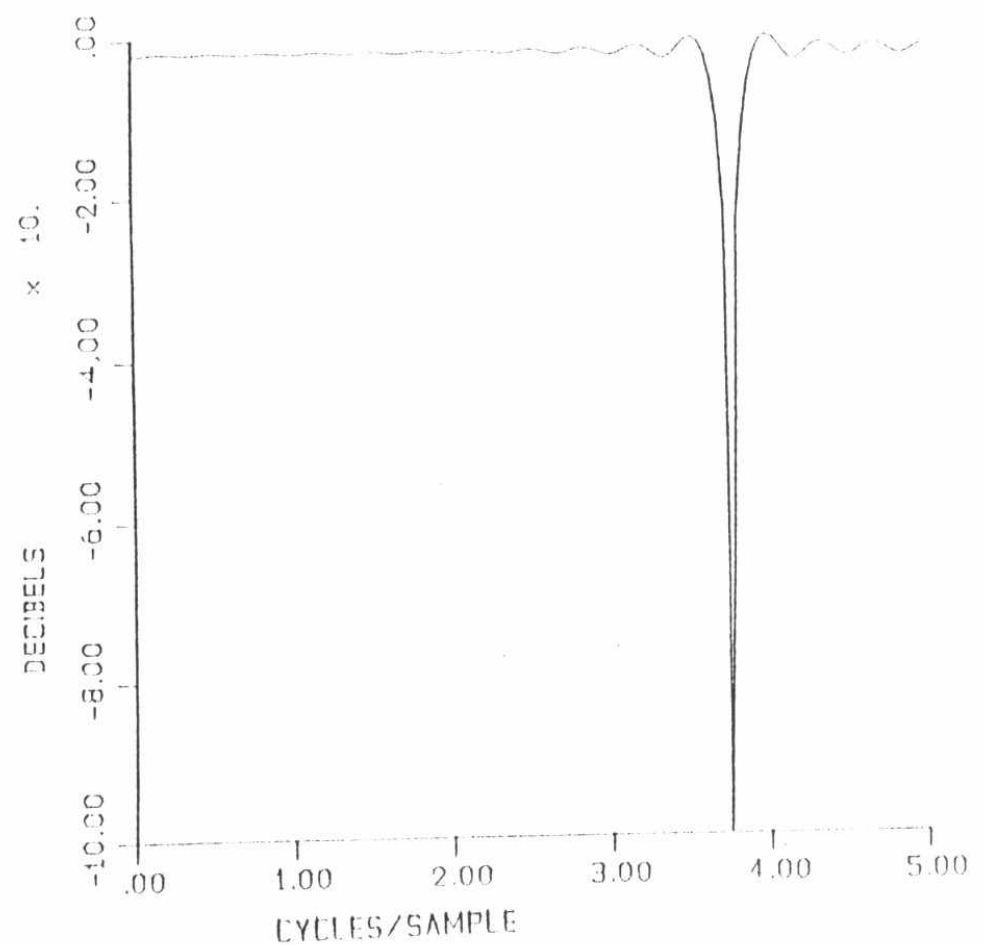

Fig. 1 Narrow-band Notch Filter Design $L=61, F_{p}=F_{s}=0.5, F_{n}=0.375$

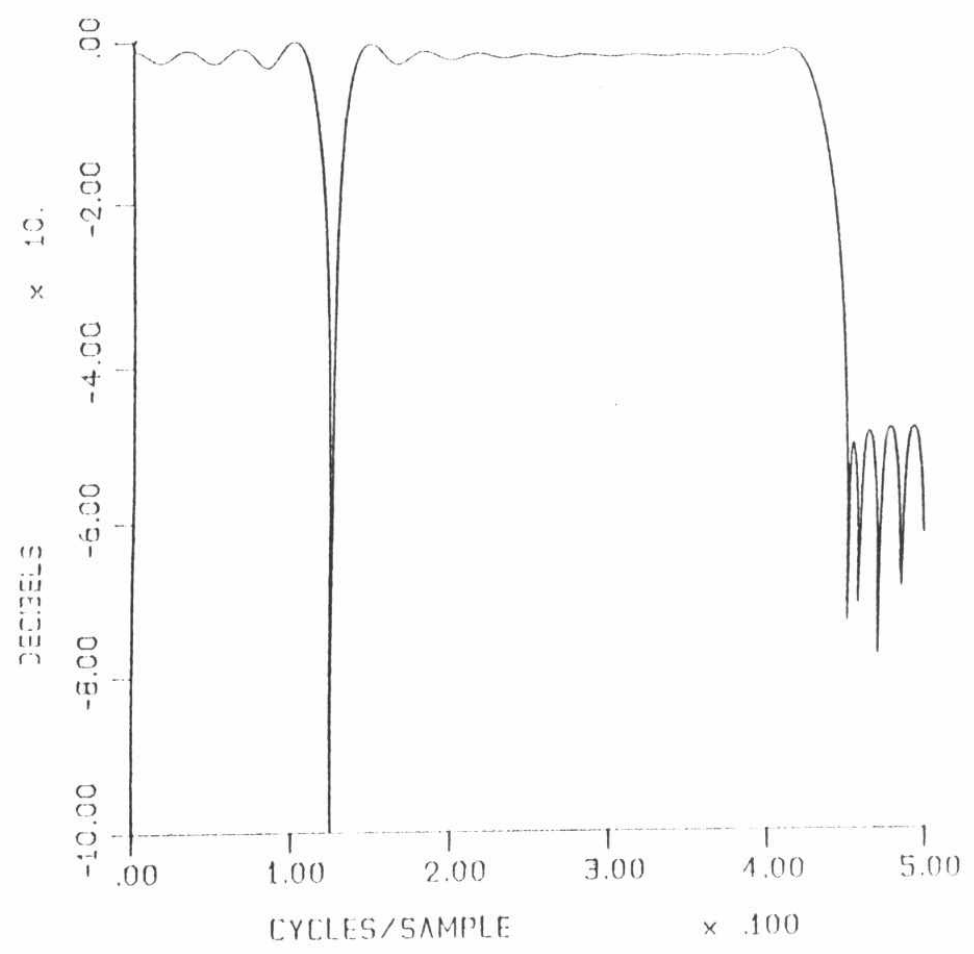

Fig. 2 Narrow-band Notch Filter Design $L=60, \mathrm{~F}_{\mathrm{p}}=0.4, \mathrm{~F}_{\mathrm{s}}=0.45, \mathrm{~F}_{\mathrm{n}}=0.125$ 